\title{
26-10 Fab-digoxin complex: Affinity and specificity due to surface complementarity
}

(x-ray crystallography/antibody-antigen complex/antibodies/anti-digoxin)

\author{
Philip D. Jeffrey*†, Roland K. Strong $¥ \S$, Larry C. Sieker*ף, Chieh Ying Y. Chang*, \\ Robert L. Campbell $\ddagger \|$, Gregory A. Petsko $¥ * *$, Edgar Haber ${ }^{* \dagger} \dagger$, Michael N. Margolies $\ddagger \ddagger$, \\ AND STEVEN SHERIFF*\$§
}

*Bristol-Myers Squibb Pharmaceutical Research Institute, P.O. Box 4000, Princeton, NJ 08543-4000; ${ }^{\ddagger}$ Department of Chemistry, Massachusetts Institute of Technology, Cambridge, MA 02139; and ¥¥Department of Surgery, Massachusetts General Hospital, Boston, MA 02114

Communicated by David R. Davies, June 24, 1993

\begin{abstract}
We have determined the three-dimensional structures of the antigen-binding fragment of the anti-digoxin monoclonal antibody $26-10$ in the uncomplexed state at $2.7 \AA$ resolution and as a complex with digoxin at $2.5 \AA$ resolution. Neither the antibody nor digoxin undergoes any significant conformational changes upon forming the complex. Digoxin interacts primarily with the antibody heavy chain and is oriented such that the carbohydrate groups are exposed to solvent and the lactone ring is buried in a deep pocket at the bottom of the combining site. Despite extensive interactions between antibody and antigen, no hydrogen bonds or salt links are formed between 26-10 and digoxin. Thus the 26-10-digoxin complex is unique among the known three-dimensional structures of antibody-antigen complexes in that specificity and high afinity arise primarily from shape complementarity.
\end{abstract}

Digoxin (digoxigenin tridigitoxoside; Structure I) inhibits the $\mathrm{Na}^{+}, \mathrm{K}^{+}$-ATPase and is used in treatment of congestive heart failure (1). Digoxin is a cardenolide-type steroid with an $\alpha, \beta$-unsaturated lactone ring attached at C-17 and three $\beta(1 \rightarrow$ 4)-D-glycoside-linked digitoxoses attached at $\mathrm{O}-3$. It is a relatively large and rigid hapten, whose internal degrees of freedom are limited to rotations about the lactone-cardenolide bond (C-17 to C-20; Structure I) and about the digitoxose

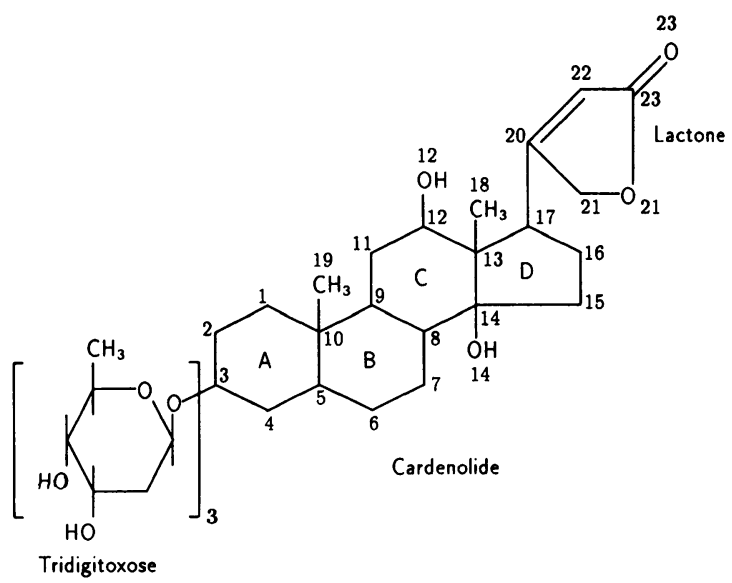

Structure I

linkages (2). Many digoxin congeners of known chemical structure are available, including several with high-resolution crystal structures (2). These analogues vary as to the substituents on the steroid rings, the number and type of attached carbohydrate groups, and the saturation of the lactone ring.

The publication costs of this article were defrayed in part by page charge payment. This article must therefore be hereby marked "advertisement" in accordance with 18 U.S.C. $\$ 1734$ solely to indicate this fact.
Anti-digoxin antibodies have been used to measure serum digoxin levels as a guide to therapy and to treat otherwise fatal digoxin intoxication (3). Anti-digoxin antibodies complexed with structurally distinct digoxin congeners are also an excellent model system to study the structural basis of antibody specificity.

The antibody $26-10$ (IgG2a, $\kappa$ light chain) is a murine monoclonal antibody that exhibits high affinity $\left(10^{10} \mathrm{M}^{-1}\right)$ for digoxin and varying specificity for digoxin congeners (4-6). Both the intact 26-10 antibody and the 26-10 Fab (antigenbinding fragment) reverse lethal digoxin toxicity in guinea pigs (7). Spontaneous and site-directed mutants of 26-10 have been generated $(6,8,9)$. The specificities of $26-10$ and its mutants have been characterized for a wide range of digoxin congeners $(6,8,9)$.

We report the three-dimensional structures of the 26-10 Fab fragment at $2.7 \AA$ resolution and of the Fab-digoxin complex at $2.5 \AA$ resolution. $ๆ 9$

\section{MATERIALS AND METHODS}

Antibody 26-10 was obtained from A/J mice immunized with a digoxin-human serum albumin conjugate (4). Purified antibody was cleaved by papain, and the Fab was purified by using ion-exchange chromatography (10). Crystals of the 26-10 Fab-digoxin complex were grown in the presence of a 3-fold molar excess of digoxin from $16 \%$ to $18 \%(\mathrm{wt} / \mathrm{wt})$ polyethylene glycol (average $\left.M_{\mathrm{r}}=8000\right)$ with $1.7 \%$ (vol/vol) 2-methyl-2,4-pentanediol in $10 \mathrm{mM}$ sodium phosphate at $\mathrm{pH}$ 7.5 (11). The complex crystals have the symmetry of space group $P 2_{1}$, with cell dimensions $a=44.2 \AA, b=164.4 \AA, c$ $=70.0 \AA$, and $\beta=108.4^{\circ}$. There are two Fab-digoxin complexes in the asymmetric unit. The crystals were twinned

Abbreviations: CDR, complementarity determining region; $\mathrm{C}_{\mathrm{H}} \mathbf{1}$ immunoglobulin heavy-chain constant domain $1 ; \mathrm{C}_{\mathrm{L}}$, immunoglobulin light-chain constant domain; $\mathrm{V}_{\mathrm{L}}$, immunoglobulin light-chain variable domain; $\mathrm{V}_{\mathrm{H}}$, immunoglobulin heavy-chain variable domain. †Present address: Box 576, Memorial Sloan-Kettering Cancer Center, 1275 York Avenue, New York, NY 10021.

§Present address: California Institute of Technology, Division of Biology, 156-29, Pasadena, CA 91125.

TPresent address: Department of Biological Structure, SM-20, University of Washington, Seattle, WA 98195.

"Present address: Institute for Biological Sciences, National Research Council Canada, Ottawa, ON, Canada K1A 0R6.

**Present address: Rosenstiel Medical Sciences Research Center, Brandeis University, Waltham, MA 02254.

${ }^{\dagger}$ Present address: Harvard School of Public Health, 677 Huntington Avenue, Boston, MA 02115.

\$To whom reprint requests should be addressed.

१ศThe data and model coordinates of the 26-10 Fab (IIGI) and Fab-digoxin (1IGJ) crystal structures have been deposited in the Protein Data Bank, Chemistry Department, Brookhaven National Laboratory, Upton, NY 11973. 
by hemimerohedry, with the $(h, k, l)$ reflections from one lattice and the $(h, k,-h-l)$ reflections from the other being spatially overlapped and not resolvable during data collection. The twin fraction, as estimated by the method of Britton (12), was highly variable between crystals (11). Data were collected on a San Diego Multiwire area detector (13) to a maximum resolution of $2.5 \AA$ (Table 1). The structure was determined by the method of molecular replacement (15) using data that had been detwinned.

The data set used for the molecular replacement solution had an estimated twin fraction of $20 \%$ and a maximum resolution of $2.7 \AA$. This data set, after detwinning, was used for the first cycles of refinement. Subsequently, a different data set was used for the refinement due to a superior maximum resolution of $2.5 \AA$. The $2.5 \AA$ data set had an estimated twin fraction in excess of $35 \%$. The structure was refined by using the program X-PLOR (16). Since the detwinning method significantly reduced the completeness of the data, a method of refining the structure on the basis of twinned data was developed. The effects of twinning were introduced into $\left|F_{\text {calc }}\right|$, with the twin fraction estimated by least-squares minimization of the crystallographic residual, given the known twinning relationship (Table 1) (P.D.J. and S.S., unpublished results). Electron density maps were calculated using detwinned data. The program CHAIN (17) was used for electron density map inspection and structure modification. After the chain trace in the antibody-combining site was completed and most of the side chains were fitted into density, a model of digoxin (2) was fitted into a large volume of unattributed positive difference density in the combining site. The digitoxose groups were deleted since there was no clear difference density for them, but it was possible to add the first digitoxose group back into the model during refinement. No solvent molecules were incorporated into the model during refinement. The final model had low deviations

Table 1. Data collection and refinement statistics

\begin{tabular}{lcc}
\hline & 26-10 Fab-digoxin & 26-10 Fab \\
\hline & Data collection & \\
Maximum resolution, $\AA$ & 2.5 & 2.7 \\
Number of observations & 68,345 & 16,046 \\
Number of reflections & 27,121 & 7998 \\
$R$ merge on intensities*, \% & 4.8 & 5.6 \\
$\left\langle I / \sigma_{I}\right\rangle$ & 10.9 & 26.3 \\
& \multicolumn{1}{c}{ Structure refinement } & \\
Resolution range, $\AA$ & $8.0-2.5$ & $8.0-2.7$ \\
Number of reflections ${ }^{\dagger}$ & 25,135 & 7326 \\
Number of atoms & 6652 & 3299 \\
Completeness of data ${ }^{\ddagger}, \%$ & 80.0 & 61.0 \\
$R$ value (uncorrected $)^{8}, \%$ & 24.3 & 17.7 \\
$R$ value (corrected) $\%, \%$ & 17.4 & \\
Estimated twin fraction & $\| .358$ & \\
rms $\Delta$ bond length, $\AA$ & 0.013 & 0.014 \\
rms $\Delta$ bond angle & $3.2^{\circ}$ & $3.3^{\circ}$ \\
rms $\Delta$ dihedrals & $28.1^{\circ}$ & $28.3^{\circ}$ \\
rms $\Delta$ impropers & $1.4^{\circ}$ & $1.3^{\circ}$ \\
Coordinate error**, $\AA$ & 0.25 & 0.30 \\
\hline
\end{tabular}

$\Delta$, Change in.

${ }^{*} \Sigma_{i}\left(\Sigma_{j}\left|\mathrm{I}_{i j}-\left\langle\mathrm{I}_{i}\right\rangle\right|\right) / \Sigma_{i}\left\langle I_{i}\right\rangle$ for $j$ observations of each unique reflection $i$. $\dagger|F|>1 \sigma_{F}$.

$\neq$ Number of reflections used in refinement as a fraction of the number of theoretically observable unique reflections.

$\S \Sigma|| F_{\text {obs }}|-k| F_{\text {calc }} \| / \Sigma\left|F_{\text {obs }}\right| ; F_{\text {obs }}$ uncorrected for twinning and $F_{\text {calc }}$ calculated from model.

IUncorrected $F_{\text {obs }} ; F_{\text {calc }}$ corrected for twinning using $F_{\text {calc }}^{\prime}:\left|F(h)_{\text {calc }}^{\prime}\right|$ $=\left((1-f) \cdot\left|F(\mathbf{h})_{\text {calc }}\right|^{2}-f\left|F\left(\mathbf{h}^{\prime}\right)_{\text {calc }}\right|^{2}\right)^{1 / 2}$, where $f$ is the estimated twin fraction, $h$ is the miller index $(h, k, l)$, and $h^{\prime}$ is $(h, k,-h-l)$.

"Estimated by least-squares minimization (P.D.J. and S.S., unpublished results)

**Luzzati (14). from ideal stereochemistry and a residual of 0.171 (corrected for twinning) for reflections in the range 8.0-2.5 $\AA$ with $|F|>$ $1 \sigma_{F}$ (Table 1).

Crystals of uncomplexed 26-10 Fab grew in the presence of a 3-fold molar excess of digoxin and $1.5 \%$ (wt/vol) formamide from $40 \%$ polyethylene glycol (average $M_{\mathrm{r}}=200$ ) in $40 \mathrm{mM}$ imidazole- $\mathrm{HCl}$ at $\mathrm{pH}$ 7.0. The crystals have the symmetry of space group $P 2_{1}$, with cell dimensions $a=43.8 \AA, b=89.3$ $\AA, c=59.2 \AA$, and $\beta=95.9^{\circ}$ with one Fab in the asymmetric unit, as reported (10). The uncomplexed crystal form was not twinned. We were unable to grow the uncomplexed Fab crystals in the absence of digoxin. Data were collected on a Siemens area detector to a maximum resolution of $2.7 \AA$ (Table 1). The structure was determined by molecular replacement using the model of the 26-10-digoxin complex with digoxin deleted, split into the immunoglobulin light-chain variable domain $\left(V_{L}\right)$ /heavy-chain variable domain $\left(V_{H}\right)$ and immunoglobulin light-chain constant domain $\left(C_{L}\right)$ /heavychain constant domain $1\left(C_{H} 1\right)$ fragments. Single clear peaks were observed for both the $V_{L} / V_{H}$ [signal/noise ratio $(s / n)$ $>1.43$ ] and $C_{L} / C_{H} 1(s / n>1.27)$ fragments in the "fast rotation function" (18) using the program MERLOT (19). Molecular translations were determined by using the program BRUTE (20), which yielded clear solutions for the Fv fragment (which consists of the $V_{L}$ and $V_{H}$ domains; $s / n>1.25$ ) and the combined translation search $(\mathrm{s} / \mathrm{n}>1.25)$. The molecular replacement solution yielded a correlation coefficient of 0.524 on $|F|^{2}$ for data in the 6-4 $\AA$ range.

The uncomplexed structure was refined using rigid body, energy minimization, and simulated annealing protocols using the program X-PLOR (16). Solvent molecules were not incorporated into the model during refinement due to the relatively low resolution and incompleteness of data. The final model had low deviations from ideal stereochemistry and a residual of 0.177 for reflections in the range 8.0-2.7 $\AA$ with $|F|>1 \sigma_{F}$ (Table 1).

Molecular surfaces and surface areas for the models were calculated by using the program MS (21) with a $1.6 \AA$ probe radius and extended atom van der Waals radii (22). Pairwise contacts were determined by using the method of Sheriff $e t$ al. (23) but using extended atom radii (22). Numbering of residues and designations of framework regions and complementarity determining regions (CDRs) are according to Kabat et al. (24).

The data and model coordinates of the 26-10 Fab and Fab-digoxin crystal structures have been deposited in the Protein Data Bank (25).

\section{RESULTS}

Uncomplexed Structure. Crystals of the uncomplexed Fab were obtained from crystallization solutions that contained excess digoxin. The excess hapten and the high affinity of 26-10 for digoxin would be expected to give rise to crystals of Fab-digoxin complex rather than the uncomplexed Fab. However, the electron density maps did not show convincing difference density for digoxin, in contrast to that observed during refinement of the 26-10-digoxin complex. When a model of the digoxin complex was refined against the uncomplexed Fab data set, the average $\mathrm{B}$ factor for digoxin was $41 \AA^{2}$, which is significantly greater than that of the combining site ( $12 \AA^{2}$ for residues in contact with digoxin). Since the crystal apparently contains uncomplexed Fab, either the effective concentration of digoxin in the crystallization medium must have been reduced, perhaps due to precipitation, or the affinity is reduced similar to that seen with phosphocholine binding to crystallized McPC603 (26).

No ordered electron density was observed for the N-terminal residue of the $\mathrm{V}_{\mathbf{H}}$ domain and the $\mathrm{C}$-terminal residues of the $\mathrm{C}_{\mathrm{L}}$ domain in the uncomplexed Fab. Two additional 
residues were added into density extending from the $\mathrm{C}$ terminus of $\mathrm{C}_{\mathrm{H}} 1$ as alanine residues, but there was no interpretable side-chain density for these residues. Other regions with poorly defined electron densities and higher $B$ factors were residues $\mathrm{H} 25-\mathrm{H} 31$ in $\mathrm{V}_{\mathrm{H}}, \mathrm{H} 53-\mathrm{H} 56$ in CDR $\mathrm{H} 2$, and $\mathrm{H} 128-\mathrm{H} 137$ in $\mathrm{C}_{\mathrm{H}} 1$.

Complexed Structure. Digoxin is bound at the center of the combining site in 26-10, between the $V_{L}$ and $V_{H}$ domains

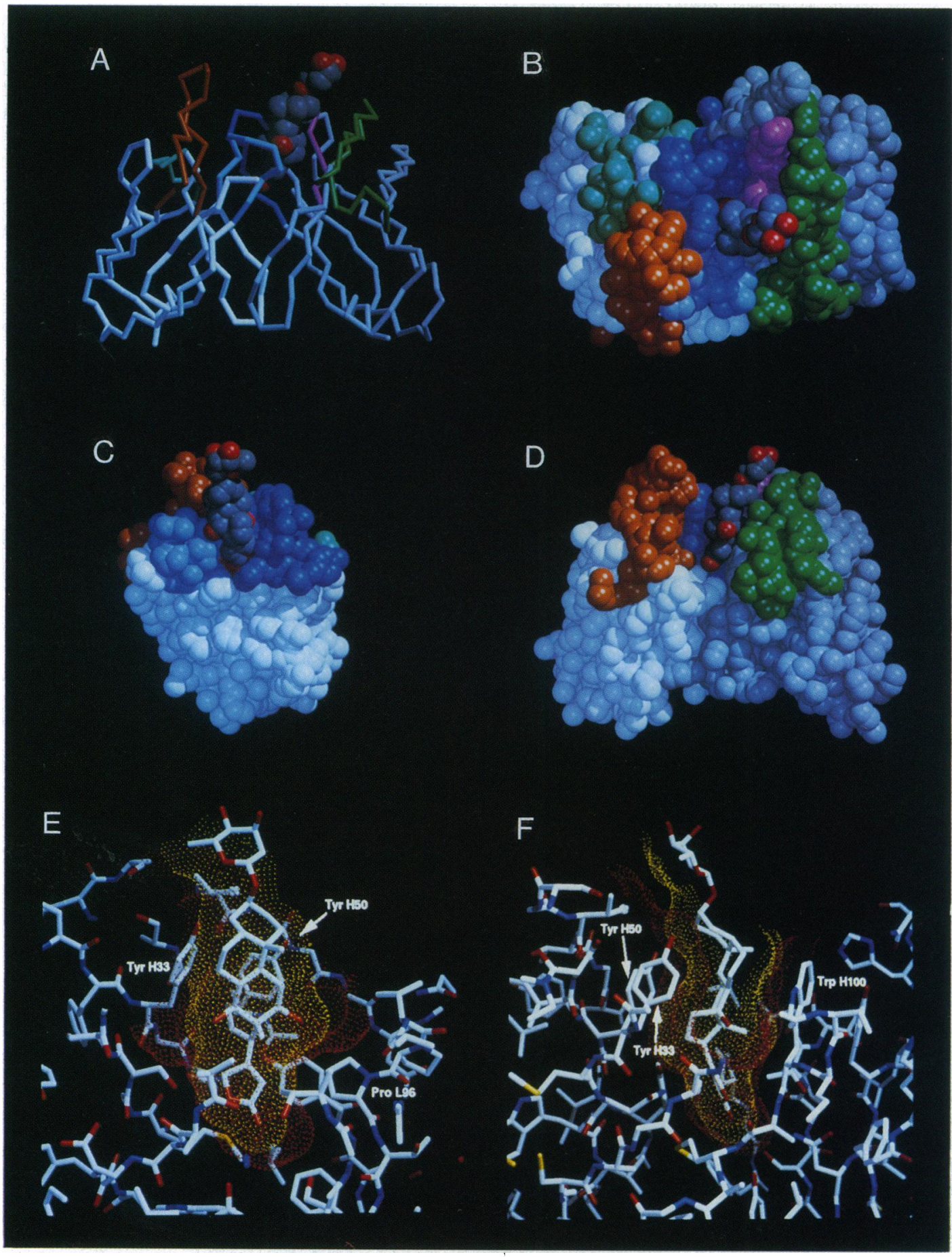

FIG. 1. (A) Schematic representation of the variable regions of the 26-10-digoxin complex, with $C^{\alpha}$ atoms connected by rods. The $V_{L}$ domain is shown in white, the $V_{H}$ domain is shown in gray, and digoxin is shown as a space-filling atomic representation. Digoxin color coding is dark gray for carbon atoms and red for oxygen atoms. The CDRs are represented as follows: L1, orange; L2, pale green; L3, pale blue; H1, purple; $\mathrm{H} 2$, green; H3, blue. (B) Space-filling representation of the 26-10-digoxin complex looking approximately down the interdomain pseudo-dyad axis of the Fv toward digoxin. Same color scheme as in $A$ is used. $(C)$ Cut-away view of the complex with all of the $V_{H}$ domain except $C D R$ $\mathrm{H} 3$ deleted. The view is approximately perpendicular to the pseudo-dyad axis, looking from $\mathrm{V}_{\mathrm{H}}$. (D) Cut-away view of the complex with CDR $L 3$ and the first two residues of $V_{L}$ removed. The view is approximately perpendicular to the pseudo-dyad axis looking from CDR $L 3$. (E) Dot surface representation of the complex as seen from $V_{L}$. The red dot surface represents the molecular surface of $26-10$ atoms buried in the complex. The yellow dot surface represents the molecular surface of digoxin atoms buried in the complex. Tyr-H47 is immediately to the left of Pro-L96. $(F)$ Dot surface representation of the complex as seen from CDR H3. This figure was generated by the program RASTER3D written by David Bacon. 
(Fig. 1A). The long axis of digoxin (from the 0-3 atom to the C-17 atom) is approximately parallel (inclined by $22^{\circ}$ ) to the pseudo 2-fold axis that interrelates the $V_{L}$ and $V_{H}$ domains (Fig. $1 A$ and $B$ ). The lactone ring is buried deepest within the combining site, and the digitoxoses are exposed to solvent (Fig. $1 C$ and $D$ ). The surface complementarity between antibody and hapten is closest at the lactone and steroid D rings and decreases toward the periphery of the binding site. Correspondingly, the atomic B factors for digoxin are lowest at the lactone ring $\left(10 \AA^{2}\right)$ and increase toward the digitoxose $\left(49 \AA^{2}\right)$. The mean $B$ factor for the lactone ring atoms is comparable to that for the 26-10 residues in the combining site that contact digoxin (13 $\AA^{2}$ ). Digoxin is sandwiched between the aromatic rings of Tyr-H33, Tyr-H50, and Trp-H100 (Fig. $1 D$ and $F$ ), but the complementarity is much less close in other regions: there are significant gaps between the surfaces in the complex around the hydroxyl groups at positions 12 and 14 on digoxin (Fig. $1 C$ and $E$ ).

As with the uncomplexed Fab, the $\mathrm{N}$ terminus of $\mathrm{V}_{\mathrm{H}}$ and the $\mathrm{C}$ terminus of $\mathrm{C}_{\mathrm{L}}$ were disordered. The same regions that were disordered in the uncomplexed Fab were disordered in the complex.

When the individual domains from two 26-10-digoxin complexes in the asymmetric unit are compared, the root-meansquare (rms) deviations on superimposing $\mathrm{C}^{\alpha}$ atoms in each domain were $0.5 \AA$ for $V_{L}, 0.8 \AA$ for $V_{H}(0.6 \AA$ excluding the disordered $\mathrm{H} 25-\mathrm{H} 31$ loop), $0.6 \AA$ for $\mathrm{C}_{\mathrm{L}}$, and $0.9 \AA$ for $\mathrm{C}_{\mathrm{H}} 1$ (0.6 $\AA$ excluding the disordered H127-H137 loop). Superimposing the two copies of digoxin gave an rms deviation of $0.2 \AA$ on all steroid ring carbon atoms (C-1 to $\mathrm{C}-17)$.

Differences Between Complexed and Uncomplexed Fab. No significant conformational changes are observed in the combining site between the complexed and uncomplexed Fab. The largest conformational change in the CDRs is in a region at the top of CDR H3 (residues H97-H99) that does not make contact with digoxin in the complex and that is involved in a crystal contact in the uncomplexed Fab structure. Residues H97-H99 have higher average B factors than the rest of CDR $\mathrm{H} 3$ in both complexed and uncomplexed structures. The residues in CDR $\mathrm{H} 3$ that make contact with digoxin (Table 2) show no significant deviations between the complexed and uncomplexed Fabs. The rms deviations on superimposing $\mathrm{C}^{\alpha}$ atoms from the Fab domains of the uncomplexed and the first of the two independent 26-10-digoxin complexes are $0.5 \AA$ for $\mathrm{V}_{\mathrm{L}}, 0.7 \AA$ for $\mathrm{V}_{\mathrm{H}}(0.6 \AA$ excluding the disordered $\mathrm{H} 25-\mathrm{H} 31$ region), $0.5 \AA$ for $C_{L}$, and $0.7 \AA$ for $C_{H} 1$. The differences in $V_{L} / V_{H}$ and $C_{L} / C_{H} 1$ pairings between complexed and uncom- plexed forms are less than $2.5^{\circ}$, compared to less than $1.0^{\circ}$ between the two complexes.

Conformational Changes in the Hapten. The position of the lactone ring of digoxin in the complex is flipped by $180^{\circ}$ about the C-17-C-20 bond, relative to the small-molecule crystal structure of digoxin (2). The orientation of the lactone is the same as observed in a number of cardiac glycoside crystal structures (2). The close complementarity around the lactone ring makes the complex specific for only one of the two possible lactone conformations. No electron density is seen for the second and third (outermost) carbohydrate groups of digoxin. No other significant conformational changes are observed in the antigen. The rms deviation for all carbon atoms in the steroid rings between the digoxin in 26-10digoxin and the structure of Go et al. (2) is $0.3 \AA$, which is comparable to the rms differences between the two copies of the complex (see above).

Interactions Between 26-10 and Digoxin. The pairwise contacts between 26-10 and digoxin in the complex are summarized in Table 2. There are no hydrogen bonds or charged group interactions between 26-10 and digoxin: all the contacts are nonpolar. Sixty-one pairwise contacts involve 10 antibody residues from four CDRs and one framework region and 19 digoxin atoms from all four steroid rings and the lactone. Additionally, the Fab residues His-L27d and Asn-L34 from CDR L1, the framework residue Tyr-L36, and the digitoxose group on digoxin are partially buried on complexation. CDR $\mathrm{L} 2$ is not directly involved in the binding of digoxin. CDR H3 makes the most extensive interactions with the hapten, including the side chain of Trp- $\mathrm{H} 100$ that extends between digoxin and CDR L1. In addition to the CDRs, the framework residue Tyr-H47 makes contacts with the hapten, and framework residue Tyr-L36 is partially buried on complex formation. Residue Tyr-H47 is a conserved Trp in $93 \%$ of mouse antibodies (24), but Trp could not be accommodated in the complex without disrupting the complementarity between the Fab and digoxin and without eliminating the hydrogen bond between the side chains of Asn-H35 and Tyr-H47. The majority of the pairwise contacts between Fab and hapten (Table 2) involve four aromatic side chains from the Fab (Tyr-H33, Tyr-H47, Tyr-H50, and Trp-H100; 36 of 62 contacts) and the lactone and D rings of digoxin (48 of 61 contacts). The monodigitoxose does not make any contacts with the Fab, although $26 \AA^{2}$ of its molecular surface is buried in the complex (Table 2).

\section{DISCUSSION}

The three-dimensional structure of the 26-10-digoxin complex allows us to qualitatively explain the fine specificity of

Table 2. Contacts between Fab and hapten

\begin{tabular}{|c|c|c|c|c|c|c|c|c|c|}
\hline Region & $\begin{array}{l}\text { Contacting } \\
\text { residues }\end{array}$ & $\begin{array}{l}\text { Pairwise } \\
\text { contacts }\end{array}$ & $\begin{array}{c}\text { Buried } \\
\text { area, } \\
\AA^{2}\end{array}$ & Digoxin region & Region & $\begin{array}{l}\text { Contacting } \\
\text { atoms }\end{array}$ & $\begin{array}{l}\text { Pairwise } \\
\text { contacts }\end{array}$ & $\begin{array}{c}\text { Buried } \\
\text { area, } \\
\AA^{2}\end{array}$ & Fab region \\
\hline \multicolumn{5}{|c|}{ Fab to hapten } & \multicolumn{5}{|c|}{ Hapten to Fab } \\
\hline CDR L1 & & 0 & 5 & & Digitoxose & & 0 & 26 & \\
\hline CDR L3 & Thr-L91, Pro-L96 & 9 & 92 & $\begin{array}{l}\text { Ring D, } \\
\text { lactone }\end{array}$ & Ring A & $\mathrm{C}-2, \mathrm{C}-3, \mathrm{C}-4$ & 4 & 36 & CDRs $\mathrm{H} 1, \mathrm{H} 2$ \\
\hline CDR H1 & Tyr-H33, Asn-H35 & 13 & 55 & $\begin{array}{l}\text { Rings A, C, D; } \\
\text { lactone }\end{array}$ & Ring B & C-6, C-7, C-19 & 8 & 36 & CDRs H1, H3 \\
\hline FR2 $V_{H}$ & Tyr-H47 & 6 & 19 & $\begin{array}{l}\text { Ring D; } \\
\text { lactone }\end{array}$ & Ring C & $\mathrm{C}-12,0-12$ & 3 & 37 & CDRs L3, H2 \\
\hline CDR H2 & Tyr-H50 & 6 & 74 & Rings A, C, D & Ring D & $\begin{array}{l}\text { O-14, C-15, C-16, } \\
\text { C-17, C-18 }\end{array}$ & 17 & 75 & $\begin{array}{l}\text { CDRs L3, H1, H2, } \\
\text { H3; FR2 V }\end{array}$ \\
\hline & $\begin{array}{l}\text { Ser-H95, Trp-H100, } \\
\text { Ala-H100a, Met-H100b }\end{array}$ & 26 & 126 & $\begin{array}{l}\text { Rings B, D; } \\
\text { lactone }\end{array}$ & Lactone & $\begin{array}{l}\text { C-20, C-21, O-21 } \\
\text { C-22, C-23, O-23 }\end{array}$ & 27 & 78 & $\begin{array}{l}\text { CDRs L3, H1, H3; } \\
\text { FR2 V }\end{array}$ \\
\hline Total & & 59 & 383 & & Total & & 59 & 274 & \\
\hline
\end{tabular}

Calculation of pairwise contacts and buried surface area is described in Materials and Methods. Noncontacting 26-10 residues contribute 79 $\AA^{2}$ of the total buried surface area. Noncontacting digoxin atoms contribute $39 \AA^{2}$ of the total buried surface area. FR2, framework region 2 . CDRs H1-H3 refer to CDRs 1-3 in the immunoglobulin heavy chain; CDRs L1 and L3 refer to CDRs 1 and 3 in the light chain. 
26-10 for many of the digoxin congeners whose binding affinity has been measured (6). The 26-10 antibody does not recognize the presence or absence of the digitoxose groups in digoxin, and the affinities for the mono-, bis- and tridigitoxose forms are identical. The lack of recognition of the carbohydrate corresponds to the limited interactions between the digitoxose and Fab in the complex and the disorder of the second and third digitoxose groups. However, the 26-10 antibody recognizes the presence and type of carbohydrate groups on digoxin congeners with substituents at C-16 and C-12 that have reduced binding affinities for 26-10 (6). The complex structure does not explain the recognition of carbohydrate in these cases, although it is possible that the orientation of the glycoside in the binding site may be slightly different, allowing greater interaction between the carbohydrate and the Fab.

The 26-10 antibody binds digitoxigenin (which lacks the $12 \beta-\mathrm{OH}$ of digoxigenin) with equal affinity to digoxigenin. Thus the $12 \beta-O H$ is not a major determinant of fine specificity, despite the two contacts made between the $12 \beta-\mathrm{OH}$ in digoxin and Pro-L96 (Table 2). The $12 \beta-\mathrm{OH}$ is only partially accessible to solvent in the complex, and it is possible that the energy gained from interactions between antibody and antigen is offset by the necessity of partially desolvating this group on forming the complex.

The close complementarity between $26-10$ and digoxin at the 16 position in the D-ring of digoxin causes 26-10 to recognize substituents at $C-16$. The $26-10$ antibody binds gitoxin $(16 \beta-O H) 5$-fold less well than digoxin (6). The small decrease in affinity suggests that the complex is able to rearrange slightly in this region, and interactions between the $\mathrm{OH}$ and the side chain of residue Asn-H35 may play a role in limiting the affinity loss. Larger substituents at C-16 would cause more disruption of the combining site, and congeners with formyl and acetyl groups at C-16 have markedly reduced affinities (6).

The crystal structures of Fab in complex with antigen and hapten (reviewed in ref. 27) have shown that antigen recognition is achieved by a combination of shape complementarity, hydrogen bonding, and charged group complementarity. In contrast to the structures of these complexes, and despite the high affinity constant for digoxin $\left(10^{10} \mathrm{M}^{-1}\right)$, there are no hydrogen-bond or charged group interactions between 26-10 and digoxin. Thus specificity and affinity must arise solely from shape complementarity. Nevertheless, there are polar groups on the Fab and the hapten within the combining site. The polar groups are stabilized either by hydrogen bonds within the Fab or by accessibility to bulk solvent. The only polar groups that are not stabilized by interactions with solvent or other polar residues are the lactone ring 0-21 and 0-23 oxygen atoms of digoxin. Although there are potential hydrogen-bond donors in the proximity of $0-21$, none are closer than $3.5 \AA$. The only neighbor of $0-21$ that has an acceptable geometry for hydrogen bonding is Ser-H95 ${ }^{\gamma}$, which already makes a $2.9 \AA$ hydrogen bond with the side-chain amide oxygen of Asn-H35 but is $3.5 \AA$ away from 0-21. Atom 0-23 also makes no hydrogen bonds, although the more distant side-chain atoms of Tyr-L36 OH (3.9 $\AA)$ and Asn-L34 $\mathrm{N}^{\delta 2}(4.2 \AA)$ may have some role in stabilizing the partial negative charge on $0-23$ in what appears to be a quite hydrophobic environment.

In common with other antibody-antigen complexes (27), the complementarity between the solvent-accessible surfaces of digoxin and 26-10 is imperfect, even in regions that are in contact. However, there are no cavities buried in the complex that are large enough to contain a solvent (water) molecule. All of the volumes that are large enough to contain solvent are accessible to bulk solvent (in particular the regions around $0-12$ and $0-14$ on digoxin). There is no electron density in the combining site that is compatible with a well-ordered water molecule making bridging hydrogen bonds between antibody and hapten, and none of the polar groups on residues making contacts with the hapten have an additional ordered water molecule bound. The imperfect complementarity is sufficient to exclude water from the interface between antibody and antigen but may reduce the overall energetic contribution of nonbonded interactions, which decay rapidly with distance. This suggests that the displacement of water ordered at the surface of digoxin, rather than dispersion forces, is the dominant term in the high affinity of 26-10 for digoxin. The rigidity of the hapten (2) and the aromatic rings in the combining site of the Fab may enhance this effect, as fewer degrees of freedom are lost on immobilizing the hapten than would be the case for a more flexible antigen. The hydrogen-bonding network in the Fab may further reduce the mobility of smaller side chains in the combining site. If the displacement of ordered water in the combining site is the cause of the high affinity of 26-10 for digoxin, in the same way as the hydrophobic effect in protein folding, then we would predict a large entropic contribution to the free energy of binding when the thermodynamics of hapten binding by the antibody are measured.

We thank Drs. Joel Schildbach, Robert Bruccoleri, and Jiri Novotny for helpful discussions and Dr. Martha Ludwig for the use of her data collection facility. This work was supported in part by National Institutes of Health Grants PO1-HL19259 and RO1HL47415.

1. Smith, T. W. (1988) N. Engl. J. Med. 318, 358-365.

2. Go, K., Kartha, G. \& Chen, J. P. (1980) Acta Crystallogr. Sect. B Struct. Sci. 36, 1811-1819.

3. Smith, T. W., Haber, E., Yeatman, L. \& Butler, V. P., Jr. (1976) N. Engl. J. Med. 294, 797-800.

4. Mudgett-Hunter, M., Margolies, M. N., Ju, A. \& Haber, E. (1982) J. Immunol. 129, 1165-1172.

5. Mudgett-Hunter, M., Anderson, W., Haber, E. \& Margolies, M. N. (1985) Mol. Immunol. 22, 477-488.

6. Schildbach, J. F., Panka, D. J., Parks, D. R., Jager, G. C., Novotny, J. Herzenberg, L. A., Mudgett-Hunter, M., Haber, E., Bruccoleri, R. E. \& Margolies, M. N. (1991) J. Biol. Chem. 266, 4640-4647.

7. Lechat, P., Mudgett-Hunter, M., Margolies, M. N., Haber, E. \& Smith, T. W. (1984) J. Pharmacol. Exp. Ther. 229, 210-213.

8. Schildbach, J. F., Near, R. I., Bruccoleri, R. E., Haber, E., Jeffrey, P. D., Novotny, J., Sheriff, S. \& Margolies, M. N. (1993) Protein Sci. 2, 206-214.

9. Schildbach, J. F., Near, R. I., Bruccoleri, R. E., Haber, E., Jeffrey, P. D., Ng, S.-C., Novotny, J., Sheriff, S. \& Margolies, M. N. (1993) J. Biol. Chem., in press.

10. Rose, D. R., Seaton, B. A., Petsko, G. A., Novotny, J., Margolies, M. N., Locke, E. \& Haber, E. (1983) J. Mol. Biol. 165, 203-206.

11. Strong, R. K. (1990) Dissertation (Harvard Univ., Cambridge, MA).

12. Britton, D. (1972) Acta Crystallogr. Sect. A Found. Crystallogr. 28, 296-297.

13. Xuong, N.-H., Freer, S. T., Hamlin, R., Nielsen, C. \& Vernon, W. (1978) Acta Crystallogr. Sect. A Found. Crystallogr. 34, 289-296.

14. Luzzati, V. (1952) Acta Crystallogr. 5, 802-810.

15. Brünger, A. T. (1991) Acta Crystallogr. Sect. A Found. Crystallogr. 47, 195-204.

16. Brünger, A. T. (1990) X-PLOR (Yale Univ., New Haven, CT), Version 2.1

17. Sack, J. S. (1988) J. Mol. Graphics 6, 224-225.

18. Crowther, R. A. (1972) in The Molecular Replacement Method, ed Rossmann, M. G. (Gordon \& Breach, New York), No. 13, pp. 171-178.

19. Fitzgerald, P. M. D. (1988) J. Appl. Crystallogr. 21, 273-278.

20. Fujinaga, M. \& Read, R. J. (1987) J. Appl. Crystallogr. 20, 517-521.

21. Connolly, M. L. (1983) J. Appl. Crystallogr. 16, 548-558.

22. Gelin, B. R. \& Karplus, M. (1979) Biochemistry 18, 1256-1268.

23. Sheriff, S., Hendrickson, W. A. \& Smith, J. L. (1987) J. Mol. Biol. 197, 273-296.

24. Kabat, E. A., Wu, T. T., Perry, H. M., Gottesman, K. S. \& Foeller, C. (1991) Sequences of Proteins of Immunological Interest (National Institutes of Health, Bethesda, MD).

25. Bernstein, F. C., Koetzle, T. F., Williams, G. J. B., Meyer, E. F., Jr., Brice, M. D., Rodgers, J. R., Kennard, O., Shimanouchi, T. \& Tasumi, M. (1977) J. Mol. Biol. 112, 535-542.

26. Rudikoff, S., Potter, M., Segal, D. M., Padlan, E. A. \& Davies, D. R. (1972) Proc. Natl. Acad. Sci. USA 69, 3689-3692.

27. Wilson, I. A. \& Stanfield, R. L. (1993) Curr. Opin. Struct. Biol. 3, 113-118. 